\title{
Estudio preliminar de índices ecocardiográficos que permitan optimizar la indicación de resonancia cardíaca en pacientes con Tetralogía de Fallot reparada.
}

\author{
Myriam Ferreiro ${ }^{1}$, Lida Toro ${ }^{1}$, Marcelo Andia ${ }^{2}$, Pamela Zelada ${ }^{1}$, Alex Alcántara ${ }^{1}$, María Elisa Castillo ${ }^{1,2}$, \\ Rosa Lagos ${ }^{1}$, Sandra Bareño ${ }^{1}$, Sergio Uribe ${ }^{1,2}$ \\ 1 Complejo Hospitalario Dr. Sótero del Río, \\ 2 Departamento de Radiología y Centro de Imágenes Biomédicas, Escuela de Medicina, Pontificia \\ Universidad Católica de Chile.
}

Introducción: La Tetralogía de Fallot reparada (TOFr) a largo plazo evoluciona frecuentemente con insuficiencia pulmonar (IP). La resonancia magnética cardíaca (RMC) juega un rol fundamental en la indicación de recambio valvular pulmonar destinada a evitar las complicaciones de la IP.

Objetivo: El objetivo de este trabajo es buscar qué índice(s) ecocardiográfico(s) permite(n) identificar a los pacientes con TOFr que tienen VD dilatado y fracción de eyección (FE) disminuida en la RMC.

Método: En 20 pacientes (9 mujeres, 8-25 años, promedio 15,8 años) con TOFr se realizó ecocardiograma (ECO) y RMC en el Hospital Sótero del Río. Por ECO se obtuvieron mediciones de excursión sistólica máxima anular tricuspídea (TAPSE), onda s tisular, aceleración miocárdica isovolumétrica (IVA), y grado de IP (leve, moderado o severo). Por RMC se midieron la FE, volumen fin de diástole (VFD) del VD y fracción de regurgitación de la arteria pulmonar (FR). Se realizó un análisis estadístico multivariado.

Resultados: No se obtuvo ninguna correlación entre algún parámetro de ECO versus VFD o FE del VD.
Sin embargo, se encontró un modelo basado en el TAPSE e IP que en nuestros pacientes pudo predecir el VDF del VD con un $\mathrm{r} 2=0.6$. (VDF-VD $=8.60 *$ TAPSE + $36.19 * \mathrm{IP}-77.213)$. Al comparar la IP con la FR se encontró que la ecografía puede distinguir correctamente aquellos casos que tienen IP leve de severa $(\mathrm{p}<0.001)$, o moderada de severa $(\mathrm{p}=0.004)$, pero no puede diferenciar con significancia estadística aquellos casos de IP leve de moderada $(\mathrm{p}=0.272$ ).

Conclusión : En este estudio preliminar se encontró un modelo estadístico basado en el TAPSE e IP que podría ser útil en la selección de pacientes que son derivados para estudio con RMC. La estimación del grado de IP medida por ECO es un parámetro fácil de obtener, sin embargo no siempre es informado en forma categórica (leve, moderado o severo). Si bien este estudio se debe validar en un mayor número de pacientes, éste nos indica la relevancia de algunos parámetros ecocardiográficos que deberían incluirse siempre en el protocolo de evaluación de pacientes con TOFr.

Palabras claves: Tetralogía de Fallot, ecocardiografía, resonancia nuclear magnética. 


\section{Echocardiographic indexes help predict the need for nuclear magnetic resonance evaluation of patients with repaired Tetralogy of Fallot}

Background: Pulmonary insufficiency (PI) frequently appears long-term after repair of Tetralogy of Fallot (TOFr). Cardiac magnetic resonance (CMR) plays a fundamental role in the indication of pulmonary valve replacement, in order to avoid complications of PI. However, CMR is a scarce and expensive resource in our reality, which is why its indication must be optimized.

Aim: The objective of this work is to find echocardiographic indices to identify patients with TOFr with dilated $\mathrm{RV}$ and reduced ejection fraction $(\mathrm{EF})$

Method: Images from echocardiograms (ECHO) and CMR in 20 patients ( 9 women, 8 -25 years of age, average 15,8 years old) with TOFr were retrospectively reviewed. From ECHO images we obtained measurements for tricuspid annular plane systolic excursion (TAPSE), tissular $\mathrm{s}$ wave, isovolumetric acceleration (IVA), and severity of PI (mild, moderate, or severe). From CMR images, we measured EF, end diastolic volume (EDV) of the RV, and regurgitant fraction (RF) of the pulmonary artery. We performed a multivariate sta- tistical analysis to explore the relation between $\mathrm{ECHO}$ parameters and CMR findings

Results: No correlation was found between individual ECHO parameters and EDV or RV EF. However, we did find a model based on the TAPSE and PI that was able to predict the EDV of the $\mathrm{RV}$ with an $\mathrm{r} 2=0.6$. (FDV-RV $=8.60 *$ TAPSE $+36.19 * \mathrm{PI}-77.213)$. After comparing the PI with RF, we found that that echocardiography could correctly distinguish cases with mild PI from those with severe PI $(\mathrm{p}<0.001)$, or moderate from severe $(\mathrm{p}=0.004)$, but not mild from moderate PI Conclusion: In this preliminary study, we found a statistical model based on the TAPSE and PI which could be useful in the selection of patients with TOFr that are referred to CMR. While this study needs to be validated on a greater number of patients, it indicates the relevance of some echocardiographic parameters, which should always be included in the evaluation of patients with TOFr.

Keywords: Cardiac magnetic resonance imaging, Echocardiography, Tetralogy of Fallot.

\section{Introducción}

La Tetralogía de Fallot es la cardiopatía cianótica más frecuente. ${ }^{1}$ La cirugía reparadora tiene excelentes resultados a corto plazo, sin embargo, en las últimas décadas se ha reconocido que estos pacientes requieren un seguimiento de por vida, dado el alto índice de complicaciones a largo plazo. ${ }^{2,3,4}$ De ellas, la más frecuente es la insuficiencia pulmonar (IP), que lleva a dilatación y disfunción del ventrículo derecho (VD) y se relaciona con muerte súbita. ${ }^{5}$ Aunque la ecocardiografía es un método ampliamente disponible para el diagnóstico y seguimiento de estos pacientes, tiene serias limitaciones en la evaluación del ventrículo derecho., ${ }^{\mathbf{6}, 7}$ Estas limitaciones incluyen, entre otras, la geometría del VD, la posición en el tórax y la disposición de las fibras musculares del VD. ${ }^{8}$ Se ha demostrado que la resonancia magnética cardíaca (RMC) es una técnica que permite una evaluación adecuada del volumen y función del VD. Por ello se ha convertido en el patrón de referencia en la indicación de recambio valvular pulmonar, destinado a evitar las complicaciones asociadas a la insuficiencia pulmonar en pacientes con Tetralogía de Fallot reparada (TOFr). ${ }^{9}$ Sin embargo, la RMC en nuestro medio es un recurso escaso y costoso por lo que se debe optimizar su indicación. Existen varios reportes en la literatura de nuevos índices ecocardiográficos que intentan estimar el volumen, la función ventricular derecha, el grado de insuficiencia pulmonar y su correlación con RMC.

El objetivo de este trabajo es buscar qué índice(s) ecocardiográfico(s) permite(n) identificar a los pacientes con TOFr que presenten dilatación significativa de VD y/o fracción de eyección disminuida en RMC.

\section{Método}

Pacientes: Se analizaron retrospectivamente las ecocardiografías y RMC de pacientes con TOFr, realizadas en el Hospital Sótero del Río, entre enero del 2011 y junio del 2012. Veinte pacientes (9 mujeres, con un promedio 
de 15,8 años y un rango entre 8-25 años) con TOFr tenían ecocardiograma y RMC, con un período entre exámenes de 4 meses en promedio (rango 0.8-9,5 m). En este período los pacientes no fueron sometidos a cateterismos intervencionales ni cirugías. Se excluyeron los pacientes con marcapaso y se obtuvo el consentimiento informado de los pacientes o sus padres.

Ecocardiografía: La ecocardiografía 2D fue realizada usando un ecocardiógrafo Vivid 7 Dimension con transductores de frecuencias adecuadas para el tamaño de los pacientes y con grabación simultánea de electrocardiograma. Las imágenes digitales fueron transferidas a una estación de trabajo para análisis posterior (ECHO PAC PC Dimension). Las medidas ecocardiográficas analizadas fueron la excursión sistólica máxima anular tricúspidea (TAPSE), la velocidad sistólica peak en la inserción lateral de la tricúspide (onda s), la aceleración miocárdica isovolumétrica (IVA) y el grado de insuficiencia pulmonar.

El TAPSE fue obtenido en modo $\mathrm{M}$ en visión de 4 cámaras, con el cursor en la pared lateral del anillo tricuspídeo. ${ }^{10,11}$ La onda s ${ }^{12,13}$ y el IVA ${ }^{14}$ se obtuvieron con Doppler tisular en visión de 4 cámaras, con el cursor en la pared lateral del anillo tricúspideo. La velocidad miocárdica máxima durante la sístole se registró a un barrido de $100 \mathrm{~mm} / \mathrm{seg}$. El IVA se calculó dividiendo la velocidad máxima durante la contracción isovolumétrica por el tiempo que toma en alcanzarla (tiempo de contracción isovolumétrica) ${ }^{\mathbf{1 4}}$.

El grado de IP fue evaluado usando Doppler color. Se clasificó en severa, si se observó flujo diastólico retrógrado en las ramas de la arteria pulmonar, moderada si el flujo diastólico retrógrado se observa sólo en el tronco de la arteria pulmonar, o leve si la IP se observa sólo en el tracto de salida del VD. ${ }^{15}$

Resonancia Magnética Cardíaca: La RMC fue realizada en un resonador $1.5 \mathrm{~T}$ (Philips Achiva, Best, The Netherlands), usando un protocolo estandarizado para pacientes con TOFr. El protocolo incluyó localizadores, secuencias de cine con una secuencia de gradientes balanceados (b-SSFP) en las siguientes vistas: eje largo del ventrículo izquierdo y derecho, 4 cámaras, tractos de salida de ambos ventrículos y de cada rama pulmonar. Además, se adquirió una serie de cines en ejes cortos para cuantificar la función cardíaca y flujos en la Aorta, Arteria pulmonar y sus ramas. El protocolo se realizó sin sedación. A partir de estas imágenes se midieron la fracción de eyección (FE), volumen fin de diástole (VFD) del VD y fracción de regurgitación (FR) de la arteria pulmonar en el software viewforum (Philips, Best, The Netherlands).

Análisis Estadístico: Se realizó un análisis estadístico multivariado analizando cuál o cuáles parámetros ecocardiográficos se correlacionaban con el VFD y/o FE del VD obtenidos por RMC. Además, se estudió la correlación entre el grado de IP medida por ECO y la FR por RMC. El análisis se realizó con el software estadístico SPSS.

\section{Resultados}

En la Tabla 1 se muestran los valores promedios medidos por ecocardiograma y resonancia magnética.

El resultado del estudio estadístico demostró que no se obtuvo correlación entre ningún parámetro individual de ECO versus VDF o FE del VD medido por RMC. Sin embargo, el análisis multivariado encontró un modelo basado en el TAPSE e IP que pudo predecir en nuestros pacientes, el VDF del VD con un $\mathrm{r} 2=0.6$ (Figura 1 ):

\begin{tabular}{|c|c|c|}
\hline & Promedio & DE \\
\hline $\begin{array}{l}\text { TAPSE } \\
\text { IVA } \\
\text { Onda s } \\
\text { FE } \\
\text { VFD_VD }\end{array}$ & $\begin{array}{l}14.3 \\
182 \\
8.2 \\
56.6 \\
121.8\end{array}$ & $\begin{array}{l}2.1 \\
65.6 \\
2.0 \\
7.8 \\
39.8\end{array}$ \\
\hline
\end{tabular}

TAPSE: Excursión anular sistólica máxima del anillo tricúspideo; IVA: aceleración miocárdica isovolumétrica; FE: fracción de eyección; VFD_VD: volumen final de diástole del ventrículo derecho

Figura 1: Correlación entre el volumen del ventrículo derecho medido por RMC versus el estimado a partir de los parámetros de ecocardiografía.

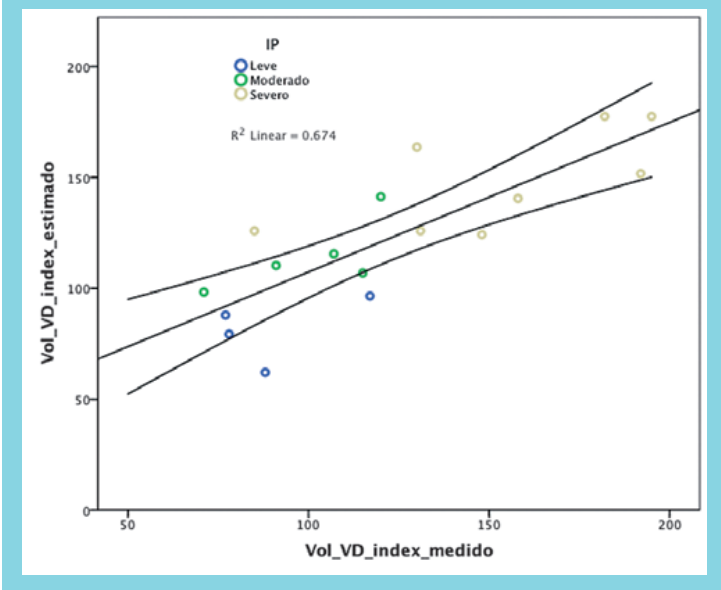


VDF_VD $=8.60 *$ TAPSE + 36.19*IP -77.213, donde los valores asignados a la insuficiencia pulmonar fueron $(0,1,2$ para insuficiencia leve, moderada y severa respectivamente).

Al comparar la IP con la FR se encontró que la ecocardiografía puede identificar correctamente aquellos casos que tengan IP leve de severa $(\mathrm{p}<0.001)$, o moderada de severa $(\mathrm{p}=0.004)$, pero no puede distinguir con significancia estadística aquellos casos de IP leve de moderada $(\mathrm{p}=0.272)$.

\section{Discusión}

La ecografía 2D no ha demostrado ser eficiente en la estimación de volúmenes de VD al compararla con RMC, ${ }^{16}$ sin embargo en este estudio preliminar se encontró un modelo estadístico basado en la medición de TAPSE y grado de IP, que podría ser útil en la selección de pacientes que serán derivados para estudio con RMC.

Se ha demostrado que la elección del momento adecuado para el recambio de la válvula pulmonar es relevante en el pronóstico funcional del VD de estos pacientes.

En un paciente asintomático con TOFr, la decisión de recambio valvular pulmonar debe considerar el VFD de VD, la fracción de eyección y el grado de insuficiencia pulmonar, entre otros factores.

Distintos reportes muestran que si el recambio valvular se hace cuando el VFD del VD es mayor a $150 \mathrm{ml} / \mathrm{m} 2$, la remodelación post cirugía puede no normalizar el volumen del VD. Por otra parte, hay que evitar el recambio precoz de la válvula, dada su vida media limitada ${ }^{17}$.

La mayoría de los trabajos que intentan estimarel volumen del VD lo hacen en base a cálculos de área, lo que implica una visualización adecuada de los bordes del endocardio de todo el VD. Sin embargo, esto es difícil en pacientes adolescentes o adultos con mala ventana acústica y con dilatación de VD. El modelo que proponemos está basado en mediciones fáciles y reproducibles, poco dependientes de la ventana acústica. ${ }^{\mathbf{1 0 - 1 4}}$
Además, otros estudios han demostrado que la medición del flujo diastólico reverso por doppler color y/o pulsado en el tronco y ramas de la arteria pulmonar se correlaciona adecuadamente con la FR medida por RMC. 18

En nuestro trabajo, la ecocardiografía 2D nos permitió distinguir con claridad la IP leve de severa y la moderada de severa. Es una técnica fácil y rápida de obtener que debería realizarse e informarse dirigidamente en todos los pacientes con TOFr.

Índices como el TAPSE, onda s e IVA se han usado en la evaluación de la función ventricular derecha. Se plantean como útiles debido a su capacidad de medir función, independiente de la geometría del VD y han demostrado alguna correlación con la FE medida por RMC 11, 12, 19 . Para TAPSE y onda s existen valores $\mathrm{Z}$ publicados para niños sanos ${ }^{10,13}$ y se han descrito valores disminuidos en pacientes con cardiopatías congénitas. $\mathbf{1 1 , 1 2}$

En nuestro trabajo, sin embargo no se evidencia correlación de ninguno de estos índices con la FE medida por RMC, esto debido probablemente al bajo tamaño muestral.

Si bien este estudio se debe validar en un mayor número de pacientes, planteamos el uso de índices fáciles de realizar, que consumen poco tiempo y que podrían orientar en la elección de pacientes con TOFr que requieren una RMC. Las limitaciones de nuestro estudio es que es retrospectivo e incluye un número limitado de pacientes. Además, el tiempo entre la ecocardiografía y la RMC fue prolongado, lo que podría influir en los resultados. No se realizó un análisis intra o inter observador sobre la variabilidad de las mediciones ecocardiográficas y de RMC.

En conclusión nosotros proponemos un modelo estadístico que integra índices ecocardiográficos basados en el TAPSE e IP que podría ayudar a optimizar el momento en que a los pacientes con TOFr deben ser derivados para estudio con RMC. 


\section{Referencias:}

1. SHINEBOURNE EA, BABU-NARAYAN SV, CARVALHO JS. Tetralogy of Fallot: from fetus to adult. Heart 2006; 92: 1353-9.

2. NORGAARD MA, LAURIDSEN P, HELVIND M, PETTERSSON G. Twenty-to-thirty- seven-year follow-up after repair for Tetralogy of Fallot. Eur J Cardiothorac Surg 1999; 16: 125-30.

3. POME G, ROSSI C, COLUCCI V, PASSINI L, MORELLO M, TAGLIERI C, et al. Late reoperations after repair of tetralogy of Fallot. Eur J Cardiothorac Surg 1992; 6: 31-5.

4. ZHAOH, MILLERDC, REITZBA, Shumway NE. Surgical repair of tetralogy of Fallot. Long-term follow-up with particular emphasis on late death and reoperation. J Thorac Cardiovasc Surg 1985; 89: 204-20.

5. GATZOULIS MA, BALAJI S, WEBBER SA, SIU SC, HOKANSON JS, POILE C, et al. Risk factors for arrhythmia and sudden cardiac death late after repair of tetralogy of Fallot: a multicenter study. Lancet 2000; 356: 975-81.

6. TULEVSKI II, ROMKES H, DODGE-KHATAMI A, VAN DER WALL EE, GROENINK M, VAN VELDHUISEN DJ, et al. Quantitative assessment of the pressure and volume overloaded right ventricle: imaging is a real challenge. Int J Cardiovasc Imaging 2002; 18: 41-51.

7. SHEEHAN F, REDINGTON A. The right ventricle: anatomy, physiology and clinical imaging. Heart 2008; 94: 1510-5.

8. MOR-AVI V, SUGENG L, LINDNER. Imaging the Forgotten Chamber: Is the Devil in the Boundary? J Am Soc Echocardiogr 2010; 23: 141-143.

9. BAUMGARTNER H, BONHOEFFER P, DE GROOT NM, DE HAAN F, DEANFIELD JE, GALIE N, et al. ESC guidelines for the management of grown-up congenital heart disease (new version 2010). Eur Heart J 2010; 31: 2915-57.

10. KOESTENBERGER M, RAVEKES W, EVERETT AD, STUEGER HP, HEINZL B, GAMILLSCHEG A, et al. Right ventricular function in infants, children and adolescents: reference values of the tricuspid annular plane systolic excursion (TAP$\mathrm{SE}$ ) in 640 healthy patients and calculation of $\mathrm{z}$ score values. Am Soc Echocardiogr 2009; 22: 715-719.

11. KOESTENBERGER M,NAGELB, RAVEKES W,EVERETT AD, STUEGER HP, HEINZL B, et al. Systolic Right Ventricular Function in Pediatric and Adolescent Patients with Tetralogy of
Fallot: Echocardiography versus Magnetic Resonance Imaging. J Am Soc Echocardiogr 2011; 24: 45-52.

12. KOESTENBERGER M, NAGEL B, RAVEKES W, AVIANA, HEINZL B, FANDL A, et al. Tricuspid Annular Peak Systolic Velocity (S) in Children and Young Adults with Pulmonary Artery Hypertension Secondary to Congenital Heart Diseases, and in Those with Repaired Tetralogy of Fallot: Echocardiography and MRI Data. J Am Soc Echocardiogr 2012; 25: 1041-9.

13. KOESTENBERGER M, NAGEL B, RAVEKES W, AVIANA, HEINZL B, CVIRN G, et al. Reference values of the tricuspid annular peak systolic velocity in healthy pediatric patients, calculation of Z-score values, and comparison to the tricuspid annular plane systolic excursion. Am J Cardiol 2012; 109: 116-21.

14. TOYONO M, HARADA K, TAMURA M, YAMAMOTO F, TAKADA G. Myocardial Acceleration During Isovolumic Contraction as a New Index of Right Ventricular Contractile Function and Its Relation to Pulmonary Regurgitation in Patients After Repair of Tetralogy of Fallot.J Am Soc Echocardiogr 2004; 17:332-7.

15. RENELLA P, ABOULHOSN J, LOHAN D, JONNALA P, MD, FINN P, et al. Two-Dimensional and Doppler Echocardiography Reliably Predict Severe Pulmonary Regurgitation as Quantified by Cardiac Magnetic Resonance. J Am Soc Echocardiogr 2010; 23: 880-6.

16. LAI WW, GAUVREAU K, RIVERA ES, SALEEB S, POWELLAJ, GEVAT. Accuracyof guideline recommendations for two-dimensional quantification of the right ventricle by echocardiography. Int J Cardiovasc Imaging 2008; 24: 691-8.

17. GEVA T. Repaired tetralogy of Fallot: the roles of cardiovascular magnetic resonance in evaluating pathophysiology and for pulmonary valve replacement decision support. J Cardiovasc Magn Reson 2011; 13: 9

18. RENELLA P, ABOULHOSN J, LOHAN DG, JONNALA P FINN JP, SATOU GM, et al. Two-dimensional and Doppler echocardiography reliably predict severe pulmonary regurgitation as quantified by cardiac magnetic resonance. J Am Soc Echocardiogr. 2010; 23: 880-6.

19. KUTTY S, ZHOU J, GAUVREAU K, TRINCADO C, POWELL AJ, GEVA T. Regional Dysfunction of the Right Ventricular Outflow Tract Reduces the Accuracy of Doppler Tissue Imaging Assessment of Global Right Ventricular Systolic Function in Patients with Repaired Tetralogy of Fallot.J Am Soc Echocardiogr 2011; 24: 637-43. 